\title{
Prevalence of cystoid macular oedema, epiretinal membrane and cataract in retinitis pigmentosa
}

\author{
Gerald Liew, ${ }^{1,2}$ Stacey Strong, ${ }^{2,3}$ Patrick Bradley, ${ }^{2}$ Philip Severn, ${ }^{2,4}$ \\ Anthony T Moore, ${ }^{2,3,5}$ Andrew R Webster, ${ }^{2,3}$ Paul Mitchell, ${ }^{1}$ Annette Kifley, ${ }^{1}$ \\ Michel Michaelides, 2,3
}

${ }^{1}$ Centre for Vision Research, Westmead Institute for Medical Research, University of Sydney, Sydney, Australia

2Department of Medical Retina, Moorfields Eye Hospital, London, UK

${ }^{3}$ University College London, Institute of Ophthalmology, London, UK

${ }^{4}$ Department of Ophthalmology, South Tees Hospital NHS Foundation Trust, Middlesbrough, UK ${ }^{5}$ Ophthalmology Department UCSF School of Medicine, San Francisco, USA

\section{Correspondence to}

Michel Michaelides, University College London, Institute of Ophthalmology, London EC1V 9EL, UK; michel.michaelides@ ucl.ac.uk

Received 22 January 2018 Revised 27 July 2018 Accepted 13 September 2018 Published Online First 5 October 2018
ABSTRACT

Background/Aims To report the prevalence of treatable complications (cystoid macular oedema, CME; epiretinal membrane, ERM and cataract) in patients with retinitis pigmentosa (RP).

Methods Consecutive patients with RP attending a tertiary eye clinic in 2012. Spectral domain-optical coherence tomography was used to determine presence of CME and ERM. Clinic records were reviewed to identify cataract and pseudophakia. Multivariable analyses adjusted for age, gender and other confounders.

Results Data are presented for 338 eyes from 169 patients. CME was present in $58.6 \%$ of patients and $50.9 \%$ of eyes and was bilateral in $73.7 \%$. ERM, cataract and pseudophakia were present in $22.8 \%, 23.4 \%$ and $11.2 \%$ eyes, respectively. In multivariable analyses, CME was associated with younger age (OR $0.81,95 \% \mathrm{Cl} 0.67$ to 0.98 ) but not with gender. Patients with ERM and cataract/pseudophakia were less likely to also have CME (OR 0.19, 95\% Cl 0.09 to 0.40 and OR 0.37, 95\% Cl 0.16 to 0.84 , respectively). CME was most prevalent in patients with autosomal-dominant inheritance (71.4\%), followed by autosomal recessive/sporadic inheritance $(58.9 \%)$ and least likely in persons with $X$ linked inheritance $(12.5 \%, p<0.001)$.

Conclusions The prevalence of treatable RP complications is high and suggests it may be clinically beneficial to screen patients with RP to identify those who may benefit from current or future interventions.

\section{INTRODUCTION}

Inherited retinal dystrophies are now one of the leading causes of irreversible blindness. ${ }^{1}$ Retinitis pigmentosa (RP, OMIM \#26800) is the most common group of inherited retinal disorders. Vision loss occurs through progressive loss of photoreceptors and development of complications such as cystoid macular oedema (CME), epiretinal membrane (ERM) and cataract. ${ }^{2}$ Treatment of these complications may improve vision ${ }^{3-5}$ even if the underlying disorder itself continues to progress. The prevalence of complications is important for planning clinical trials, power calculations and service delivery. The prevalence of CME has been reported from clinic-based surveys to range between $11 \%$ and $20 \%$ as detected by fluorescein angiography ${ }^{67}$ and between $5.5 \%$ and $49 \%$ on optical coherence tomography (OCT). ${ }^{8-13}$
The aim of this study is to report the prevalence of CME, ERM and cataract in a hospital-based sample of patients presenting to a tertiary eye hospital.

\section{METHODS}

This is a cross-section prevalence study of patients reviewed in the inherited retinal dystrophy clinics at Moorfields Eye Hospital NHS Foundation Trust, London, UK, from January 2012 to December 2012 inclusive.

Patients were included in the study if they had a consultant (MM, ATM, ARW) confirmed diagnosis of RP or rod cone dystrophy. The diagnosis of RP was based on a history of nyctalopia and evidence of peripheral visual field constriction, characteristic fundus findings on fundus examination, presence of fundus autofluorescence abnormalities such as peripheral hypoautofluorescence and central perimacular hyperautofluorescent rings and full field electroretinogram (ERG) testing in keeping with rod-cone dystrophy. ERG testing was performed according to the International Society for Clinical Electrophysiology of Vision (ISCEV) standards. ${ }^{14}$ Inheritance pattern was determined from pedigrees and RP was categorised as autosomal dominant (AD) (eg, one affected parent or child, equal gender distribution), autosomal recessive (AR) (eg, no affected parents, consanguinity, simplex cases) and X linked RP (eg, males only, no male-to-male transmission, mothers may have signs). A limited number of patients had a molecular diagnosis. We excluded patients if they had cone rod dystrophy, $A B C A 4$ retinopathy, $\mathrm{X}$ linked retinoschisis, choroideremia, paraneoplastic retinopathy or autoimmune retinopathy. Patients with other causes of CME such as diabetes, retinal vascular occlusion or uveitis were also excluded. Further details are published elsewhere. ${ }^{15}$

The Spectralis HRA + OCT with viewing module V.5.1.2.0 (Heidelberg Engineering, Heidelberg, Germany) was used to acquire autofluorescence and spectral domain-OCT (SD-OCT) images. The SD-OCT protocol used a dense horizontal linear scan centred on the fovea which covered most of the macular region between the vascular arcades. The HEYEX software interface (V.1.6.2.0; Heidelberg Engineering) was used to view and analyse images. OCT images were scrutinised for the presence of CME which was defined as the presence of hyporeflective cystic spaces on two or more consecutive macular raster scans. Presence of ERM was defined from OCT images as hyper-reflectivity of 


\begin{tabular}{|ll}
\hline Table 1 & Baseline characteristics of cohort \\
\hline Characteristics & Number or $\%$ \\
\hline Age, years (SD) & $47.1( \pm 18.4)$ \\
\hline Male (\%) & $46.8 \%$ \\
\hline Autosomal dominant (\%) & $42(24.9 \%)$ \\
\hline RP1 & 11 \\
\hline RHO & 6 \\
\hline PRPF31 & 3 \\
\hline RP11 & 3 \\
\hline PRPF8 & 2 \\
\hline RDS & 2 \\
\hline RP9 & 1 \\
\hline Unknown/not tested & 14 \\
\hline Autosomal recessive/sporadic (\%) & $112(66.3 \%)$ \\
\hline USH2A & 10 \\
\hline MYO7A & 3 \\
\hline USH1C & 2 \\
\hline CRB1 & 2 \\
\hline Unknown/not tested & 95 \\
\hline X linked (\%) & $8(4.7 \%)$ \\
\hline RPGR & 6 \\
\hline Unknown/not tested & 2 \\
\hline Unclear inheritance & $7(4.1 \%)$ \\
\hline Ausoma domint, autosomatressive and X linked & \\
\hline
\end{tabular}

Autosomal dominant, autosomal recessive and $\mathrm{X}$ linked were determined clinically, with molecular confirmation in some patients.

the innermost retinal layer with or without foveal anatomical distortion. Lens status was assessed from clinical notes and pseudophakia was confirmed from clinical or surgical records.

\section{Statistical analyses}

SAS V.9.2 (SAS, Cary, North Carolina) was used for analyses. Fisher's exact test was used for comparing categorical variables, while the Student's t-test and ANOVA were used for comparing continuous variables. Logistic regression models by subject were used to perform multivariable analyses adjusting for age, gender and other factors at the patient level. Results were considered significant at $\mathrm{p}<0.05$. OR and $95 \% \mathrm{CI}$ are provided.

\section{RESULTS}

There were 169 patients and 338 eyes included in this study. The mean age of patients was 47.1 years $( \pm 18.4)$ (table 1$)$. Males comprised $46.8 \%$ of patients. The majority of patients had autosomal recessive/sporadic inheritance and mutations in RP1 and USH $2 A$ were the most common cause identified among those patients who underwent genetic testing.

CME was present in $58.6 \%$ of patients and $50.9 \%$ of eyes. Of these patients, $73.7 \%$ had bilateral CME (table 2). ERM was present in $25.4 \%$ of patients and $22.8 \%$ of eyes and was bilateral in $79.1 \%$ patients. Cataract was present in $27.2 \%$ of patients, $23.4 \%$ of eyes and bilateral in $71.7 \%$ patients. Pseudophakia was present in $11.8 \%$ of patients, $11.2 \%$ of eyes and was bilateral in $95 \%$ of patients. Among patients with CME, 31.3\% were mild enough that they were not treated, $37.4 \%$ were currently being treated with oral acetazolamide, $29.3 \%$ with topical dorzolamide and $2 \%$ had been previously treated. The inverse association with CME prevalence and age persists after controlling for inheritance pattern.

In table 3, multivariable age-gender adjusted associations are reported. CME was associated with younger age (OR 0.81, 95\% CI 0.67 to 0.98 ) but not with gender. CME was most prevalent
Table 2 Prevalence of cystoid macular oedema, epiretinal membrane and cataract

\begin{tabular}{lll}
\hline & By patient, N (\%) & By eye, N (\%) \\
\hline All patients & 169 & 338 \\
\hline Cystoid macular oedema & $99(58.6)$ & $172(50.9)$ \\
\hline Bilateral & $73(73.7)$ & - \\
\hline Untreated & $31(31.3)$ & $53(30.8)$ \\
\hline Oral acetazolamide & $37(37.4)$ & $68(39.5)$ \\
\hline Topical dorzolamide & $29(29.3)$ & $49(28.5)$ \\
\hline Past treatment & $2(2.0)$ & $2(1.2)$ \\
\hline Epiretinal membrane & $43(25.4)$ & $77(22.8)$ \\
\hline Bilateral & $34(79.1)$ & - \\
\hline Cataract & $46(27.2)$ & $79(23.4)$ \\
Bilateral & $33(71.7)$ & - \\
\hline Pseudophakia & $20(11.8)$ & $38(11.2)$ \\
\hline Bilateral & $19(95.0)$ & - \\
\hline
\end{tabular}

in patients with $\mathrm{AD}$ inheritance $(71.4 \%$ with $\mathrm{CME}$ in at least one eye), followed by $\mathrm{AR} /$ sporadic inheritance $(58.9 \%)$ and was least likely in those with $\mathrm{X}$ linked inheritance $(12.5 \%, \mathrm{p}<0.001)$. The OR for having CME in X linked inheritance was $0.0595 \%$ CI 0.006 to 0.48$)$. Patients with ERM and cataract/pseudophakia were less likely to also have CME (OR $0.1995 \%$ CI 0.09 to 0.40 and OR $0.37,95 \%$ CI 0.16 to 0.84 , respectively).

\section{DISCUSSION}

The burden of blindness from inherited retinal dystrophies such as RP is increasing, ${ }^{1}$ due partly to the lack of effective treatments for this group of disorders. A number of sight impairing complications are associated with RP, and vision outcome may be improved when these complications are identified and treated. We report that in a hospital-based sample of 169 patients with RP (338 eyes), the prevalence of CME, ERM, cataract and pseudophakia in eyes was $50.9 \%, 22.8 \%, 23.4 \%$ and $13.6 \%$, respectively. If one eye had an RP complication, the other eye was also highly likely to be involved $(>70 \%)$. These data suggest a relatively high prevalence of potentially treatable complications and suggests it is worthwhile screening patients with RP, including with OCT, regularly to detect these conditions.

Our prevalence findings for CME are similar to the higher estimates reported by other investigators. A trial of 39 patients by Adackapara $e t \mathrm{al}^{8}$ recruited to test the efficacy of lutein supplements in RP reported a CME prevalence of $49 \%$ in patients (and $47 \%$ in eyes) at baseline. Another clinic-based study by Hajali et $a l^{10}$ of consecutive patients with RP reported a lower CME prevalence of $38 \%$ in 124 patients, of whom $27 \%$ had bilateral CME. One reason for the lower prevalence reported in the study by Hajali $e t a l^{10}$ may be the stricter definition of CME used, where more than one cystoid space was required to define CME. Similar studies using this definition have also reported lower prevalence of CME in patients, for example, that by Testa et $a l^{16}$ which reported CME prevalence of $22.9 \%$. The study by Testa $e t$ al $^{16}$ excluded patients with Usher syndrome, which comprised a large proportion of our patients with RP and may partly contribute to the lower estimate. Other studies reporting lower estimates used time-domain OCT which may have missed small cystoid spaces due to the resolution on time domain. For example, Hagiwara et $a l^{9}$ using time domain OCT reported a prevalence of macular cysts as low as 5.5\%. Studies using a similar definition to that used in our study have reported CME prevalence of $26.9 \%$ to $49 \% .^{811}$ 
Table 3 Associations with cystoid macular oedema, epiretinal membrane and cataract

\begin{tabular}{|c|c|c|c|}
\hline & Cystoid macular oedema & Epiretinal membrane & Cataract/pseudophakia \\
\hline & \multicolumn{3}{|l|}{ OR $(95 \% \mathrm{Cl})^{*}$} \\
\hline Age, per 10 years increase & 0.81 (0.67 to 0.98$)$ & $1.13(0.91$ to 1.39$)$ & 2.30 (1.68 to 3.14$)$ \\
\hline \multicolumn{4}{|l|}{ Inheritance pattern } \\
\hline AR/sporadic & 1.0 (reference) & 1.0 & 1.0 \\
\hline$A D$ & 2.00 (0.90 to 4.43$)$ & $0.93(0.40$ to 2.13$)$ & $0.41(0.16$ to 1.10$)$ \\
\hline Cystoid macular oedema & - & - & - \\
\hline Epiretinal membrane & $0.19(0.09$ to 0.40$) \dagger$ & - & - \\
\hline Cataract/pseudophakia & $0.37(0.16$ to 0.84$) \dagger$ & - & - \\
\hline
\end{tabular}

Bolded values are significant at $\mathrm{p}<0.05$

${ }^{*}$ Adjusted for age. Gender was not significantly associated in any analyses.

†Additionally adjusted for other variables significant in univariable analyses that is, age, inheritance pattern, epiretinal membrane and cataract/pseudophakia.

$A D$, autosomal dominant; $A R$, autosomal recessive; $X L, X$ linked.

We report ERM prevalence of $22.8 \%$ among eyes in our study. A wide range of ERM prevalence in eyes with RP and detected using OCT has been reported, ranging from 0.6\%, $15.6 \%$, $27.3 \%$ to $64.3 \% .^{913} 1617$ The highest prevalence was reported by Grigoropoulos et $a l^{17}$ who studied patients with advanced RP and used time domain OCT, which may account for the different estimates. Hagiwara et $a l^{9}$ reported the lowest ERM prevalence of $0.6 \%$ using time domain OCT which is less sensitive than the SD-OCT used in our study.

There are limited data on the prevalence of cataract in eyes with RP. We found $23.4 \%$ of eyes had cataract, while $13.6 \%$ were pseudophakic. This compares with a reported cataract and pseudophakia/aphakia prevalence of $36.8 \%$ and $15.4 \%$ in eyes with RP by Testa et $a l^{16}$ from an Italian population and $44.4 \%$ with posterior subcapsular cataract in a Japanese population. ${ }^{18}$ Rates of pseudophakia may not be directly comparable across different countries due to different health systems and thresholds for cataract surgery.

Few studies have reported on the associations of CME. Knowledge of associations is useful in providing insights into the underlying pathophysiology, which remains an area of active research. ${ }^{19}$ We found CME was associated with younger age, more likely in $\mathrm{AD}$ inheritance and least in X linked inheritance and less likely in the presence of ERM or cataract. That CME is less likely in older patients has been suggested, ${ }^{20}{ }^{21}$ but not previously demonstrated in clinical studies. A positive association with $\mathrm{AD}$ inheritance but negative with $\mathrm{X}$ linked inheritance has also been reported by Sandberg et al and Testa et al, ${ }^{1622}$ which Hajali et $a l^{10}$ found as well, although the result was not statistically significant in the study of Hajali et al. ${ }^{10}$ Finally, Testa et al ${ }^{16}$ have also reported that CME is less likely in patients who are pseudophakic, as in our study. These results suggest that the pathophysiological process leading to CME in RP may differ from that leading to cataract and ERM, as the conditions do not appear to frequently coexist, unlike in other retinal disorders such as diabetic retinopathy. ${ }^{23}$ Our results support the hypothesis that relatively healthy retinal tissue is required to cause CME, for example, through dysfunction of Muller cell osmoregulation, ${ }^{19}$ which would explain why younger patients and milder forms of $\mathrm{RP}$ (such as AD forms) are more likely to have CME than more severe forms (X linked RP). This would also explain the inverse association between CME and ERM as well as cataract, as the latter two conditions occur in later life, when there is less healthy retinal tissue remaining.
Strengths of this study include its moderately large sample size, systematic evaluation of CME and ERM according to a prespecified protocol and multivariable adjustment for age and other confounders. Limitations include that lens status was determined from clinical records, with variability in documentation of cataract status. Severity and type of cataract were not documented. The status of pseudophakia, however, is robust as this was checked from surgical records. We combined simplex inheritance cases with AR in our analyses, which may have included de novo mutations and introduced some misclassification into this category. This misclassification is likely to be small as the majority of simplex cases are later found to have AR inheritance. ${ }^{2}$ The prevalence estimates from this study do not apply to all patients with RP and are likely higher than the true population prevalence of RP complications. However, these estimates are clinically relevant as they derive from a sample of patients presenting to tertiary eye clinics, which is how most patients with RP are managed and where recruitment would occur for clinical trials of new therapies. The prevalence estimates reported here will be of value in power calculations and trial design.

In conclusion, we report that the prevalence of RP complications in a tertiary eye hospital setting is high, with many cases showing bilateral involvement. Younger age, AD inheritance and the absence of ERM and cataract/pseudophakia were associated with increased risk of CME. The high prevalence of treatable RP complications suggests it may be clinically beneficial for eye care professionals to screen patients with RP with SD-OCT on a regular basis to identify those who may benefit from current or future interventions.

Contributors GL, ATM, ARW and MM conceived the study idea. GL, MM, SS, PB and PS designed the study and collected data. AK and GL performed statistical analyses. GL drafted the initial manuscript. GL, SS, PB, PS, ATM, ARW, PM, AK and MM all reviewed the draft and provided critical evaluations and improvements.

Funding The work was supported by grants from NHMRC Australia, National Institute for Health Research Biomedical Research Centre at Moorfields Eye Hospital National Health Service Foundation Trust and UCL Institute of Ophthalmology, Fight For Sight (UK) and FFSMercer Fund, Moorfields Eye Hospital Special Trustees, Macular Disease Society, the Foundation Fighting Blindness (USA) and Retinitis Pigmentosa Fighting Blindness. MM is supported by an FFB Career Development Award.

Disclaimer The sponsors or funding organisations had no role in the design or conduct of this research. The authors have no proprietary or commercial interest in any materials discussed in this article.

Competing interests None declared. 
Patient consent Not required.

Ethics approval Human Research Ethics Committee of Moorfields Eye Hospital NHS Foundation Trust.

Provenance and peer review Not commissioned; externally peer reviewed.

Data sharing statement Unpublished data are available on contacting the corresponding author.

\section{REFERENCES}

1 Liew G, Michaelides M, Bunce C. A comparison of the causes of blindness certifications in England and Wales in working age adults (16-64 years), 1999-2000 with 2009-2010. BMJ Open 2014;4:e004015.

2 Hartong DT, Berson EL, Dryja TP. Retinitis pigmentosa. Lancet 2006;368:1795-809.

3 Dikopf MS, Chow CC, Mieler WF, et al. Cataract extraction outcomes and the prevalence of zonular insufficiency in retinitis pigmentosa. Am J Ophthalmol 2013;156:82-8.

4 Ikeda Y, Yoshida N, Murakami Y, et al. Long-term surgical outcomes of epiretinal membrane in patients with retinitis pigmentosa. Sci Rep 2015:5:13078.

5 Strong SA, Gurbaxani A, Michaelides M. Treatment of retinitis pigmentosa-associated cystoid macular oedema using intravitreal aflibercept (eylea) despite minimal response to ranibizumab (lucentis): a case report. Case Rep Ophthalmol 2016;7:389-97.

6 Newsome DA. Retinal fluorescein leakage in retinitis pigmentosa. Am J Ophthalmol 1986;101:354-60.

7 Pruett RC. Retinitis pigmentosa: clinical observations and correlations. Trans Am Ophthalmol Soc 1983;81:693-735.

8 Adackapara CA, Sunness JS, Dibernardo CW, et al. Prevalence of cystoid macular edema and stability in oct retinal thickness in eyes with retinitis pigmentosa during a 48-week lutein trial. Retina 2008;28:103-10.

9 Hagiwara A, Yamamoto S, Ogata K, et al. Macular abnormalities in patients with retinitis pigmentosa: prevalence on OCT examination and outcomes of vitreoretina surgery. Acta Ophthalmol 2011;89:e122-e5.

10 Hajali M, Fishman GA, Anderson RJ. The prevalence of cystoid macular oedema in retinitis pigmentosa patients determined by optical coherence tomography. Br.J.Ophthalmol 2008;92:1065-8.
11 Makiyama Y, Oishi A, Otani A, et al. Prevalence and spatial distribution of cystoid spaces in retinitis pigmentosa: investigation with spectral domain optical coherence tomography. Retina 2014;34:981-8.

12 Testa F, Melillo P, Rossi S, et al. Prevalence of macular abnormalities assessed by optical coherence tomography in patients with Usher syndrome. Ophthalmic Genet 2018;39:17-21.

13 Triolo G, Pierro L, Parodi MB, et al. Spectral domain optical coherence tomography findings in patients with retinitis pigmentosa. Ophthalmic Res 2013;50:160-4.

14 McCulloch DL, Marmor MF, Brigell MG, et al. ISCEV Standard for full-field clinical electroretinography (2015 update). Doc.Ophthalmol 2015;130:1-12.

15 Liew G, Moore AT, Webster AR, et al. Efficacy and prognostic factors of response to carbonic anhydrase inhibitors in management of cystoid macular edema in retinitis pigmentosa. Invest Ophthalmol Vis Sci 2015;56:1531-6.

16 Testa F, Rossi S, Colucci R, et al. Macular abnormalities in Italian patients with retinitis pigmentosa. Br J Ophthalmol 2014;98:946-50.

17 Grigoropoulos VG, Emfietzoglou J, Nikolaidis P, et al. Optical coherence tomography findings in patients with retinitis pigmentosa and low visual acuity. Ophthalmic Surg Lasers Imaging 2010;41:35-9.

18 Fujiwara K, Ikeda Y, Murakami Y, et al. Risk factors for posterior subcapsular cataract in retinitis pigmentosa. Invest Ophthalmol Vis Sci 2017;58:2534-7.

19 Strong S, Liew G, Michaelides M. Retinitis pigmentosa-associated cystoid macular oedema: pathogenesis and avenues of intervention. Br J Ophthalmol 2017; 101:31-7.

20 Lupo S, Grenga PL, Vingolo EM. Fourier-domain optical coherence tomography and microperimetry findings in retinitis pigmentosa. Am J Ophthalmol 2011;151:106-11.

21 Mrejen S, Audo I, Bonnel S, et al. Retinitis pigmentosa and other dystrophies. Dev Ophthalmol 2017:58:191-201.

22 Sandberg MA, Brockhurst RJ, Gaudio AR, et al. Visual acuity is related to parafoveal retinal thickness in patients with retinitis pigmentosa and macular cysts. Invest Ophthalmol Vis Sci 2008;49:4568-72.

23 Akbar Khan I, Mohamed MD, Mann SS, et al. Prevalence of vitreomacular interface abnormalities on spectral domain optical coherence tomography of patients undergoing macular photocoagulation for centre involving diabetic macular oedema. Br J Ophthalmol 2015;99:1078-81. 